\title{
Identifying Core Functional Networks and Functional Modules within Artificial Neural Networks via Subsets Regression
}

\author{
Roby Velez \\ Evolving Al Lab \\ Computer Science Dept. \\ University of Wyoming \\ rvelez@uwyo.com
}

\author{
Jeff Clune \\ Evolving Al Lab \\ Computer Science Dept. \\ University of Wyoming \\ jeffclune@uwyo.com
}

\begin{abstract}
As the power and capabilities of Artificial Neural Networks (ANNs) grow, so do their size and complexity. To both decipher and improve ANNs, we need to build better tools that help us understand their inner workings. To that end, we introduce an algorithm called Subsets Regression on network Connectivity (SRC). SRC allows us to prune away unimportant nodes and connections in ANNs, revealing a core functional network (CFN) that is simpler and thus easier to analyze. SRC can also identify functional modules within an ANN. We demonstrate SRC's capabilities on both directly and indirectly encoded ANNs evolved to solve a modular problem. In many of the cases when evolution produces a highly entangled, non-modular ANN, SRC reveals that a CFN is hidden within these networks that is actually sparse and modular. That finding will substantially impact the sizable and ongoing research into the evolution of modularity and will encourage researchers to revisit previous results on that topic. We also show that the SRC algorithm can more accurately estimate the modularity Q-Score of a network than state-of-the-art approaches. Overall, SRC enables us to greatly simplify ANNs in order to better understand and improve them, and reveals that they often contain hidden modular structures within.
\end{abstract}

\section{Keywords}

Artificial Neural Networks; Analysis; Subsets Regression; Modularity; Functional Modules

\section{INTRODUCTION}

Artificial Neural Networks (ANNs) have recently become the leading machine learning method on a wide array of challenging problems [2]. Algorithms and other analysis tools that allow researchers to better understand how individual ANNs function enable us to gain insights about them and thus further improve them $[1,6,31]$. However, understand-

Permission to make digital or hard copies of all or part of this work for personal or classroom use is granted without fee provided that copies are not made or distributed for profit or commercial advantage and that copies bear this notice and the full citation on the first page. Copyrights for components of this work owned by others than the author(s) must be honored. Abstracting with credit is permitted. To copy otherwise, or republish, to post on servers or to redistribute to lists, requires prior specific permission and/or a fee. Request permissions from permissions@acm.org.

GECCO '16, July 20 - 24, 2016, Denver, CO, USA

(C) 2016 Copyright held by the owner/author(s). Publication rights licensed to ACM ISBN 978-1-4503-4206-3/16/07 . \$ \$15.00

DOI: http://dx.doi.org/10.1145/2908812.2908839

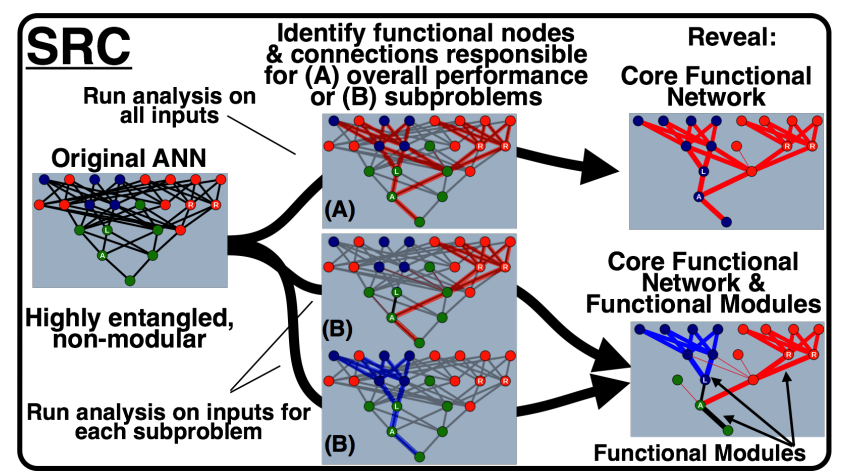

Figure 1: Identification of a Core Functional Network (CFN) and functional modules within an ANN via a new algorithm called Subsets Regression on network Connectivity (SRC). Evolutionary or learning algorithms often produce entangled, non-modular networks (left). Upper Path (A): SRC identifies nodes it deems to be functional and responsible for an ANN's overall performance and prunes the rest, revealing a fully operational, functional, yet sparser core network (CFN). Lower Path (B): SRC identifies sets of nodes (red and blue) responsible for solving different subproblems. When those sets are combined a core functional network $(\mathrm{CFN})$ is revealed, but so are functional modules; indicated by the red, blue, and black connections.

ing the inner workings of ANNs remains difficult because even small ones contain numerous components that interact in complex, non-linear ways.

This paper introduces two methods that help decipher ANNs. The first identifies nodes and connections responsible for an ANN's ability to solve an entire problem and prunes away everything else, leaving a simpler core functional network (CFN) to analyze (Fig. 1, Upper Path (A)). The second method identifies sets of nodes and connections responsible for an ANN's performance on specific subproblems and then combines those sets to reveal functional modules as well as a CFN (Fig. 1, Lower Path (B)).

\section{PREVIOUS WORK}

A CFN is a simplified version of an ANN that still performs approximately the same function. The SRC algorithm introduced in this paper creates CFNs by pruning away unimportant nodes and connections. CFNs are easier to analyze because of the reduced volume of components. We are 
unaware of any previous work that simplifies ANNs for the purpose of analysis. Instead, previous pruning techniques centered on reducing computational costs during learning and execution, or producing ANNs that generalize better to new data [1]. These pruning methods focused on identifying influential nodes and connections based on their impact on performance [13], the magnitude of weights [10], or mutual information [30].

The SRC algorithm we introduce provides a new mechanism for identifying functional modules within an ANN. Modules are clusters of highly interconnected nodes that are sparsely connected to other nodes $[6,17,26,29]$. Functional modules are modules responsible for an ANN's ability to solve specific sub-components (subproblems) of an overall problem [17]. There has been a long history of identifying, quantifying, and encouraging network modules $[6,12,21]$.

The functionality of these modules has been inferred either through visual observations $[6,12]$ or by algorithmically determining that a node somewhere in the ANN solves a known subproblem [6]. SRC provides a new, more explicit method for identifying functional modules.

$\mathrm{SRC}$ was inspired by analysis techniques performed on large natural networks that quantified the relationship between neurons in natural brains $[23,24]$ or genes in Gene Regulatory Networks [9] through correlation. With SRC, to quantify the relationship between nodes within ANNs, we implement regression, specifically subsets regression, instead of correlation. Regression is implemented instead of correlation or other analysis techniques like mutual information because regression is better equipped to analyze systems with multiple inputs. Regression will be explained in the next section, but in essence the coefficient of determination $\left(R^{2}\right)$ calculated during regression is analogous to correlation, and can quantify the relationship between nodes within an ANN.

Subsets regression, and similar techniques such as LASSO [27], have been incorporated in machine learning to reduce the dimensionality of inputs in order to ease computation and improve generalization, but have not been applied to an ANN's nodes and connections in order to analyze or simplify it. In this work the application of subsets regression on an ANN's connectivity (SRC) provides one of the first approaches to identify CFNs and functional modules, both of which can aid our understanding of ANNs.

\section{METHODS}

\subsection{Retina Problem}

To test the abilities of SRC we applied it to ANNs evolved to solve the retina problem [12] (Fig. 2). We chose the retina problem because it is a well known test domain featuring numerous experiments that investigate various strategies for producing modular ANNs [4,6, 12,28]. We tested SRC on ANNs evolved with a direct encoding to maximize performance with and without a connection cost (PCC-Direct and PA-Direct) [6], and ANNs evolved with an indirect encoding (HyperNEAT-LEO [28]) to maximize performance with and without a connection cost (PCC-Indirect and PAIndirect) [28]. Direct and indirect encodings employ different methodologies for mapping genotypes to phenotypes, and produce irregular versus regular phenotypes, respectively $[7,25]$. The addition of a connection cost during evolution can make the difference between modular and nonmodular ANNs $[6,8,11,18]$. These strategies (treatments)

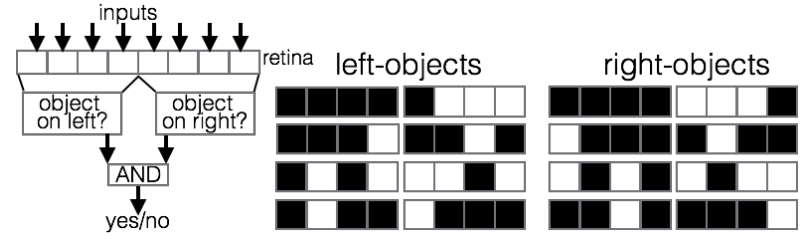

Figure 2: The Modular Retina Problem The modular retina problem is widely-used in modularity studies $[6,12]$. Networks are presented with 8-bit input patterns and must recognize the presence of "objects", which are 4-bit patterns of interest. There are left-objects, which can appear in the leftmost 4 bits of an input pattern, and right-objects, which can appear in the rightmost 4 bits. Networks must fire (output a number $>0$ ) only when both objects are present in the input. The modular solution to the retina problem involves an ANN separately solving the left-object and right-object pattern recognition tasks and then combining the output of those modules into a final AND module to produce the correct answer. Figure adapted from [12], [6], and [11].

provide an opportunity to test SRC on ANNs that look and function differently, yet still solve the same problem.

All ANNs were evolved with the Sferes2 platform [20] following the procedures and parameters from [11]. 30 runs were performed for each direct treatment and all produced perfect solutions. For the indirect treatments many more runs had to be performed in order to obtain at least 30 perfect solutions (67 for PA-Indirect and 57 for PCC-Indirect). Only perfect solutions were analyzed through SRC because they had the highest probability of possessing core functional networks (CFN) and functional modules that solved the entire retina problem or its subproblems.

\subsection{Multivariable Regression}

Subsets regression, which is at the heart of SRC, is a multivariable regression simplification technique [19]. The purpose of multivariable regression is to find a model that describes the relationship between the inputs and output of a system. When applied to an ANN, the model found describes the relationship between the signals coming into a node, also known as the predictor variables $\left(x_{1}, x_{2}, \ldots, x_{p}\right)$, and the output or activation of that node, also known as the response variable $(y)$.

In this paper, the model built by multivariable regression to map the predictor variables $\left(x_{1}, x_{2}, \ldots, x_{p}\right)$ to the response variable $(y)$ is a multilinear regression of the form:

$$
\hat{y}=\beta_{0}+\beta_{1} x_{1}+\beta_{2} x_{2}+\ldots+\beta_{p} x_{p}+I
$$

$I$ represents the 2 nd order interactions between predictors. The 2nd order interactions are the pairwise combinations of predictor variables, minus repeats and combinations of predictors with themselves. Given $p$ predictors there are $\frac{p !}{(p-1) !(2)}$ interactions.

$$
I=\beta_{12} x_{1} x_{2}+\beta_{13} x_{1} x_{3}+\beta_{23} x_{2} x_{3}+\ldots+\beta_{p-1 p} x_{p-1} x_{p}
$$

The quality of the model, which is how well the predicted value $\hat{y}$ matches the actual response variable $y$, is quantified via the coefficient of determination $\left(R^{2}\right) . R^{2}$ is a value from 0 to 1 and is analogous to correlation. Given some model, the relationship or correlation between the predictor variables and response variable can be approximated by $R^{2}$. 
In multivariable regression a system can posses many predictor variables that are not needed to describe the system's output, and can therefore be pruned to simplify the model. Subsets regression is a method to reduce the number of predictor variables and find the best subsets of predictor variables that can still satisfactorily, according to the $R^{2}$ value, describe the output of the system. To illustrate how subsets regression works the next section steps through applying subsets regression on an ANN's connectivity (SRC).

\subsection{Subsets Regression on ANN Connectivity}

Subsets regression on an ANN's connectivity (SRC) consists of three basic steps that repeat. SRC begins with a Breadth First Search (BFS) starting at the ANN's output. In this first step (1) the output node is chosen as the system of interest to which multivariable regression is applied. The activation of the output node is the output of the system and the incoming signals from other nodes are the inputs. In the second step (2) SRC calculates the individual and combinatorial contributions from the incoming signals and their effect on the output node's activation. In the third step (3), SRC selects the input or groups of inputs that are most influential, and eliminates all of the incoming connections that do not play a significant role in activating the output node. The algorithm first attempts to select single connections, then pairs of connections, then triplets, etc.

The non-influential connections are trimmed from the ANN and the BFS continues expanding nodes for which the joining connection have not been removed. A new node is selected as the current system (1), the contribution of incoming connections is calculated (2), and the most influential connections are kept while the non-influential ones are removed (3). The BFS ends once it has expanded all nodes, with the exception of the ANN's inputs nodes, that can be reached. The resulting ANN has been trimmed of all noninfluential connections and nodes revealing a core functional network (CFN). The next sections along with Figure 3 steps through the process of applying SRC to the retina ANN shown in Figure 1 (Upper Path (A)).

In the first step (1), the analysis begins at the output node o0, which is called the current response node (Fig. 3, Top). The nodes 12 and 13 have outgoing connections to o0 and are called the predictor nodes. The activations of the response and predictor nodes are the response $(y)$ and predictor variables $\left(x_{1}, x_{2}, \ldots, x_{p}\right)$ respectively, and relate back to the multivariable regression discussed previously.

In the second step (2), the predictor variables (node activations) for nodes 12 and 13 are combined into subsets $(\{12\},\{13\},\{12,13\})$. For $p$ predictor variables there are $2^{p}$ 1 subsets. Each subset of predictor variables is fed through the multilinear equation (Eq. 1), and the multivariable regression finds the model between the subset and the current response variable $y$. Once the model is built the $R^{2}$ value is calculated. The higher the $R^{2}$ value the more influential that subset of predictor variables (activation of predictor nodes) is towards the response variable (activation of response node).

For the third step (3), in order to select the smallest subset with the highest $R^{2}$, the subsets are first sorted from smallest to largest. Subsets of the same size, a tier, are then sorted again by their $R^{2}$ value from largest to smallest. For example, Figure 3 (Top) shows two tiers: tier 1 with subsets of size $1(\{12\}$ and $\{13\})$ and tier $_{2}$ with the subset of size

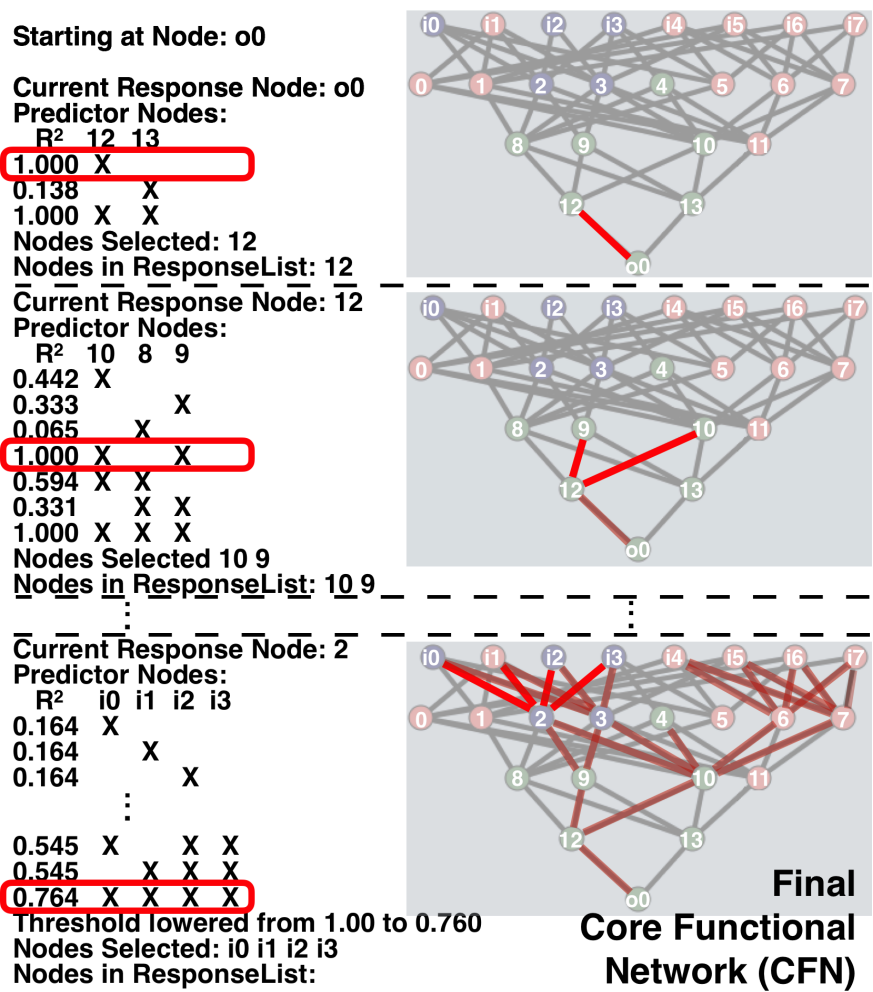

Figure 3: Subsets Regression on ANN Connectivity (SRC) Procedure See Section 3.3 for explanation.

$2(\{12,13\})$. Within tier $_{1}$ subset $\{12\}$ is higher than subset $\{13\}$ because it has a larger $R^{2}$ value, 1.000 versus 0.138 .

SRC iterates through the tiers starting at the tier with the smallest subsets, tier $_{1}$. Within a tier if the $R^{2}$ of one or more subsets satisfies a threshold, $\tau$, then those subsets are selected and the tier iteration stops. To satisfy the threshold the $R^{2}$ of a subset must be greater or equal to $\tau$. For ANNs analyzed in this paper $\tau$ starts at 1.000. In Figure 3 (Top) the subset $\{12\}$ is selected with an $R^{2}$ value of 1.000 . An $R^{2}$ of 1 means that by itself, node 12 can completely predict the activation of node o0. Node 13 is not needed and its removal simplifies the ANN without losing functionality.

While iterating through the tiers if no subsets are found that satisfy $\tau$ then $\tau$ is dropped by some amount, $\delta$, and the iteration restarts. The iteration through the tiers repeats until subsets are selected or $\tau$ becomes less than 0 . When a new response node is analyzed, $\tau$ resets back to 1 . For all ANNs analyzed in this paper $\delta$ is 0.01 .

At the end of the cycle once a subset or subsets are selected the nodes associated with those subsets, in this case node 12 , are added to the CFN and to a list of response nodes to be expanded by the BFS. Note, input nodes are not added to the response node list. The BFS picks the next node to expand, which in this case is node 12 . Node 12 is the new response node and the cycle repeats (Fig. 3, Middle).

The last cycle of SRC occurs when the BFS expands the last node in the response node list, node 2 (Fig. 3, Bottom). No subset(s) of predictor variables for the nodes, i0, i1, i2, and $i 3$, satisfies $\tau$. $\tau$ is reduced until it gets to 0.76 where the subset $\{\mathrm{i} 0, \mathrm{i} 1, \mathrm{i} 2$, and $\mathrm{i} 3\}$ in $\mathrm{tier}_{4}$ is selected. The nodes i0, i1, i2, and i3 are added to the CFN, but not to the response list because they are ANN input nodes. With no more nodes in the response list the analysis stops, revealing 


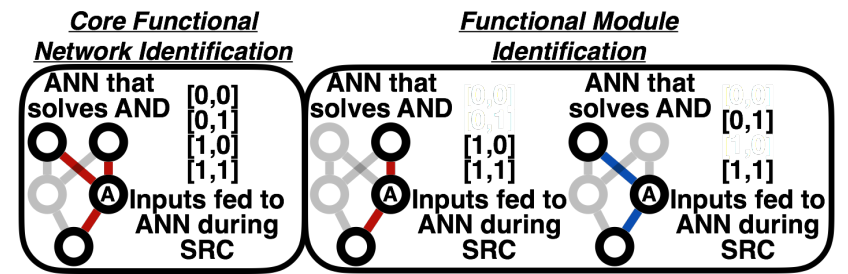

Figure 4: Illustration of how SRC isolates sets of functional nodes and connections through the variation of input patterns. Simple AND solving ANN. The specific node that solves the AND is labeled with an 'A'. Left: When all input patterns are fed to the ANN during SRC the nodes and connections responsible for solving the entire problem are found, revealing a CFN. Middle \& Right: When inputs are varied different sub-networks, responsible for different subproblems, are identified. These sub-networks can be combined (not shown) to form functional modules. See main text for full details.

a sparse, modular CFN where originally there was a highly entangled, non-modular ANN.

\subsection{Bias Nodes}

Regression, like correlation, cannot identify predictor variables that are constant. Bias nodes, which have constant outputs, are known to be very important for ANNs and should be retained during SRC. To mitigate the removal of bias nodes during SRC, any predictor node whose activation has zero variance, indicating a constant output (bias node), is deemed to be functional and retained in the CFN. These bias nodes are not added to the list of response nodes and will not be expanded by the BFS. Bias nodes are indicated by thin connections to the CFN (Fig. 1, Final CFNs).

\subsection{Functional Modules}

Figure 4 presents a simple demonstration of how SRC can identify functional modules. During SRC, if we feed all possible inputs to a simple ANN evolved to solve the AND problem, the nodes and connections that contribute to the ANNs ability to answer the overall problem are identified revealing a CFN (Fig. 4, Left). This procedure of using all the inputs patterns is demonstrated on the retina problem in Figure 1 (Upper Path (A)).

If we only feed the ANN inputs that send a ' 1 ' to the left input then the left input node is constant, it's always True (Fig. 4, Middle). During SRC the left input node is invisible to the regression and is not picked up. On the other hand the nodes and connections that encode for the right input and the AND are picked up because they are constantly changing and actively determining whether the ANN fires or not. Conversely, if we then only feed the ANN inputs that send a ' 1 ' to the right input then the right input becomes invisible and the left input and AND nodes are picked up by SRC (Fig. 4, Right).

In the case of the retina ANN, we can break up the input patterns in a similar manner. If we feed input patterns to the ANN that only contain the left-objects, then as a whole, the nodes and connections that encode the left-object (leftobject functional module) have a nearly constant output and are invisible to SRC. This is not to say that individual nodes within the left-object functional module are constant. Rather the final node(s) within the left-object functional module, which distill the left-object information and answer the subproblem, will always be on. These final left-object subproblem nodes are pruned from the ANN because SRC does not see them, and when these nodes are removed everything upstream of them goes as well. The only functional modules that are picked up by SRC are those that encode the right-object and AND subproblems (Fig. 1, Lower Path (B)) because they are changing and actively determining whether the ANN fires or not. If we feed input patterns to the ANN that only contain the right-objects the converse occurs and the functional modules responsible for the left-object and ANN subproblems are isolated.

When the sub-networks identified from feeding only leftobject or only right-object inputs are combined, the nodes common to both sets are those that encode for the AND subproblem. They are marked with black connections in the final CFN in Figure 1 (Lower Path (B)). The nodes exclusive to both sub-networks are the nodes that encode for the left-object and right-object subproblems. They are marked with red and blue connections in the final CFN in Figure 1 (Lower Path (B)).

The identification of these sub-networks through the variation of inputs patterns requires a bit more domain knowledge. The experimenter must have a sense of what input patterns cause certain nodes and connections of a ANN to be constant (invisible) or active (visible). The pay off to incorporating domain knowledge into the SRC analysis is the identification of functional modules along with the CFN.

\subsection{Knockout Analysis}

To evaluate whether the CFNs and functional modules identified by SRC posses nodes and connections responsible for overall performance or subproblems we applied a knockout analysis. For every connection in an original ANN, the ANN is rerun with that connection removed. A new fitness is obtained and the number of nodes that solve subproblems [6] is recalculated. The new fitness and number of subproblem nodes is compared to the original ANN's fitness and number of subproblem nodes to calculate a percent reduction. The percent reduction in fitness and subproblems is tabulated and binned depending on whether the removed connection was part of the CFN (functional or non-functional), was a random connection within the $\mathrm{ANN}$, or if the connection belonged to a functional module.

\section{RESULTS AND DISCUSSION}

\subsection{Core Functional Network}

To test its ability to identify CFNs, we applied the default SRC algorithm (Fig. 1, Upper Path (A)) to ANNs that solve the retina problem. Figure 5 shows examples of PA-Direct, PA-Indirect, and PCC-Indirect ANNs before and after CFN identification. PCC-Direct is omitted because its ANNs are so sparse that SRC does not prune any connections.

SRC removes non-functional connections from ANNs. We know the connections are non-functional because, with the exception of PCC-Direct, many of the ANNs lose a significant number of connections (Fig. 6, Right) yet median fitness does not drop (Fig. 6, Left). In fact when a knockout analysis is performed, the removal of a connection SRC determines to be non-functional has almost no effect on fitness, and that effect is significantly less $(p<0.001)$ than the effect of removing a random connection (Fig. 7). We can also conclude that the connections identified as functional are in- 

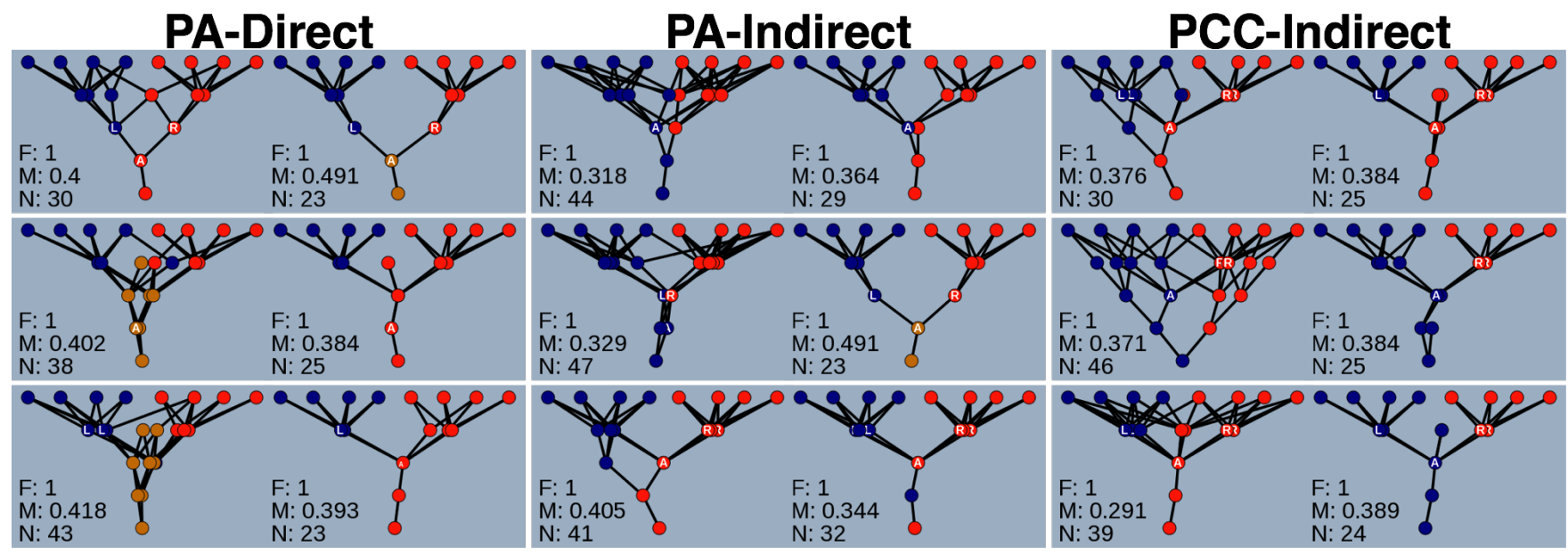

Figure 5: SRC reveals modular, core functional networks (CFNs) within networks that appear non-modular. PA-Direct, PA-Indirect, and PCC-Indirect ANNs are shown before (left of each pair) and after (right of each pair) SRC prunes nodes and connections it deems non-functional. PCC-Direct is not shown because those ANNs are already very sparse and lose no connections during SRC. ANNs are presented with optimal neuron placement (ONP) [3] to better illustrate their modular decomposition. Across different treatment encodings and objectives, many ANNs initially look entangled and non-modular. The pruning of nodes and connections, deemed non-functional by SRC, reveals simpler and more modular core functional networks (CFNs). For all ANNs shown, SRC simplification reduces the number of connections while maintaining fitness. For some of the ANNs, even though the CFN is visually more modular, a lower or almost equivalent modularity score (Q), compared to the original, is calculated revealing a potential shortcoming with the Q-Metric [16,21]. Inset text displays the fitness (F), modularity (M), and number of connections (N) for the original ANNs and simplified CFNs. Nodes or pairs of nodes that solve the retina's three subproblems - left-object, right-object, or AND - are labeled with an L, R, or A. Neurons that solve subproblems are identified as in [6]. The networks displayed were chosen to best illustrate the hidden modular structure revealed by pruning. PCC-Direct networks look qualitatively similar to the CFN networks from all three treatments shown. All ANNs and CFNs from this paper can be found at evolvingai.org/SRC and additional PCC-Direct networks from a previous study can be seen in [6].
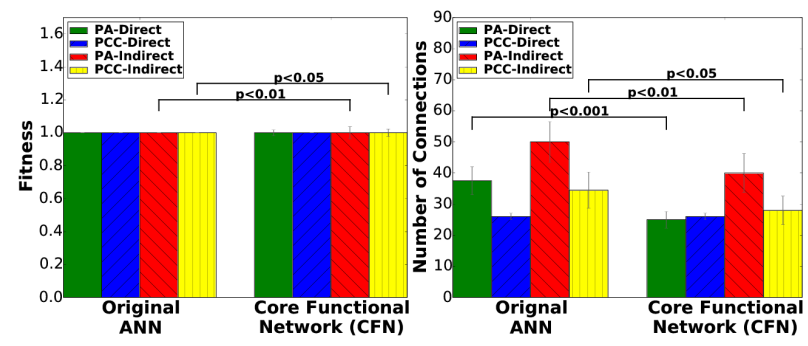

Figure 6: Median fitness and the number of connections before and after SRC simplification. For all treatments, with the exception of PCC Direct, SRC prunes a significant number of connections without lowering median fitness. For all bar plots in this paper, bars represent median values and whiskers represent $95 \%$ bootstrapped confidence interval of the median.

deed so, because on average removing one produces a drop in fitness that is significantly larger than removing a random connection (Fig. 7, $p<0.05$ ).

PCC-Direct networks lose no connections during simplification because there are no non-functional connections to prune. The inability to prune PCC-Direct ANNs without hurting performance makes sense because a connection cost pressures them to be as sparse as possible while solving the problem, therefore there should be nothing to prune.

An interesting takeaway from the CFN simplification is that for the highly entangled, non-modular treatmentsPA-Direct, PCC-Indirect, and PA-Indirect-not all connections are needed. ANN researchers commonly assume that

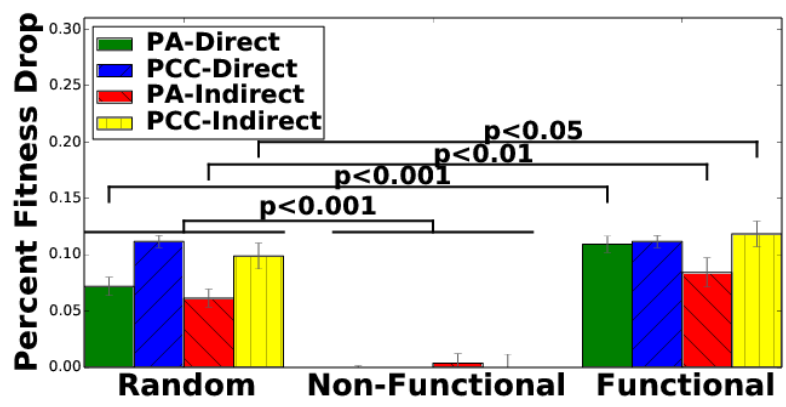

Figure 7: Knockout of functional and non-functional connections. The removal of a connection SRC deems to be non-functional has almost no impact on fitness, while the removal of a connection SRC deems to be functional severely impairs an ANN's ability to solve the problem. With the exception of PCC-Direct ANNs, connections identified by SRC as non-functional have significantly less impact on fitness than random connections $(p<0.001)$. Connections identified by SRC as functional have significantly more impact on fitness than random connections (at least $p<0.05$ ). Because SRC prunes no PCC-Direct connections, no connections are deemed non-functional and the set of connections identified by SRC as functional is the same as the set of random connections.

such highly entangled, non-modular ANNs distributively encode information throughout all connections within the network [6,12]. For all treatments save PCC-Direct, not all connections are functional (Figs. 6 and 7). 


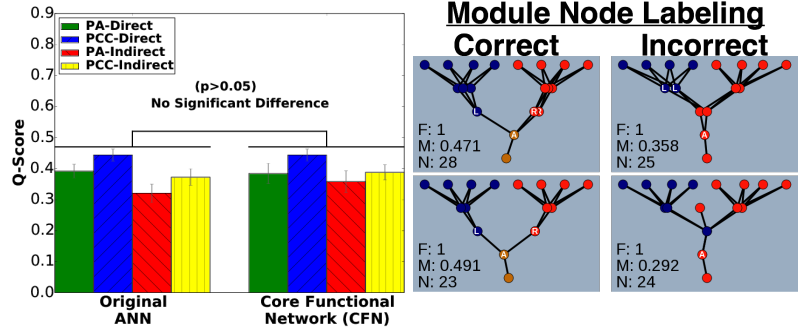

Figure 8: Q-Metric for CFNs. The Q-Metric, the state of the art in module identification, can only be calculated after a modular split is decided upon [16,22]. State-of-the-art approximations [21] can make mistakes identifying modules within ANNs resulting in inaccurately low Q-Scores. Left: Modularity (Q-Score) of treatments before and after SRC simplification. There is no significant difference $(p>0.05)$ between the original ANNs and simplified CFNs, across all treatments, even though the CFNs visually appear more modular than the original ANNs (Fig. 10). Right: Examples of CFNs with correct and incorrect module labeling (node colors). Even though all networks posses the same modular structure - a module on the left, a module on the right, and a module downstream of both - the node labeling (colors) and Q-Score for the CFNs are not consistent.

Many of the CFNs in Figure 5 look more modular after simplification, but their modularity Q-Scores do not significantly increase (Fig. 8, Left). The widely-used Q-Metric, for quantifying modularity, can only be generated for a network given a modular decomposition $[16,22]$. Finding the optimal modular decomposition that maximizes the Q-score is computationally intractable, even for small networks, so fast approximation algorithms are employed. The networks in this paper are partitioned by a state-of-the-art community detection algorithm that splits a network based on the largest eigenvalues of a modularity matrix [21]. Our results show that the state-of-art is imperfect.

All the ANNs in Figure 8 (Right) posses the same structure; a module on the left, a module on the right, and a module downstream of the left and right modules. An effective community detection algorithm should place (label) nodes into these three obvious modules, but for many ANNs the eigenvalue-based algorithm does not produce a sensible modular split (Fig. 8, Right), resulting in CFNs that are visually more modular than the original ANNs not receiving meaningfully higher Q-Scores (Fig. 8, Left).

The inconsistent labeling of nodes highlights that the eigenvalue-based approximation of the optimal modular split for the Q-Metric is imperfect. Through the application of SRC we have demonstrated another flaw or rather limitation. In Figure 5 we showed that hidden underneath highly entangled, non-modular ANNs there can exist modular structures. A community detection algorithm based on modularity maximization will never be able to discover these hidden modular structures because the Q-metric only takes into account a network's topology, not network activity, when identifying modules $[16,21]$. In the next section we show that SRC can find functional modules in the midst of highly entangled ANNs (Fig. 1, Lower Path (B)), and that those modules can serve as a better modular decomposition than the state-of-the-art approximation, yielding higher (and thus, by definition, more accurate) Q-Scores.

\subsection{Functional Modules}

Figure 10 shows a selection of CFNs created by first identifying modules deemed functional by SRC. The modules (colored connections) visually line up with the expected modular decomposition of the retina problem. Blue and red connections line up with the left-object and right-object modules, while black connections line up with the AND module (Fig. 2). Moreover, neurons that solve subproblems are found within the appropriate module (Fig. 10). SRC thus appears to be discovering functional modules. Next we quantitatively test that hypothesis via a knockout analysis.

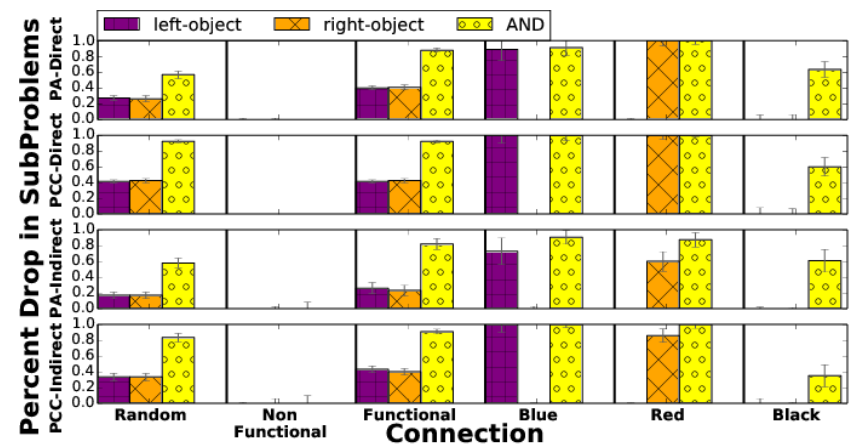

Figure 9: Percent drop in subproblems after knockout. The removal of connections within specific modules that SRC identifies as being functional disrupts an ANN's ability to solve specific subproblems, supporting the notion that the modules are indeed functional.

Figure 9 shows that a knockout of connections within the modules identified by SRC affects the CFNs ability to solve subproblems. When connections in the blue module are removed, only the left-object and AND subproblems are affected. When connections in the red module are removed, only the right-object and AND subproblems are affected. Finally, when connections in the black module are removed, only the AND subproblem is affected. The congruency with the known modular decomposition of the retina problem and the targeted influence of the modules on subproblems supports the claim that the modules identified through SRC encode those subproblems and are indeed functional.

Previously we showed that the eigenvalue based community detection algorithm can fail to find an appropriate modular split in clearly modular networks (Fig. 8, Right), resulting in lowered than expected Q-Scores (Fig. 8, Left). The functional modules found through SRC line up very well with the expected retina modules (Fig. 10). If nodes are placed into modules based on their location within the functional modules (Fig. 11, Top), the node coloring makes much more sense, and a new Functional Q-Score can be calculated. The Functional Q-Scores for all treatments are significantly higher (at least $p<0.05$ ) than their original ANN Q-Scores (Fig. 11, Bottom). Thus, given some domain knowledge to identify functional modules, SRC improves over the state-ofthe-art in approximating modular decompositions that improve our ability to accurately provide modularity Q-scores for networks, a technique that can improve analyses in all the diverse fields of network science that measure modularity. 

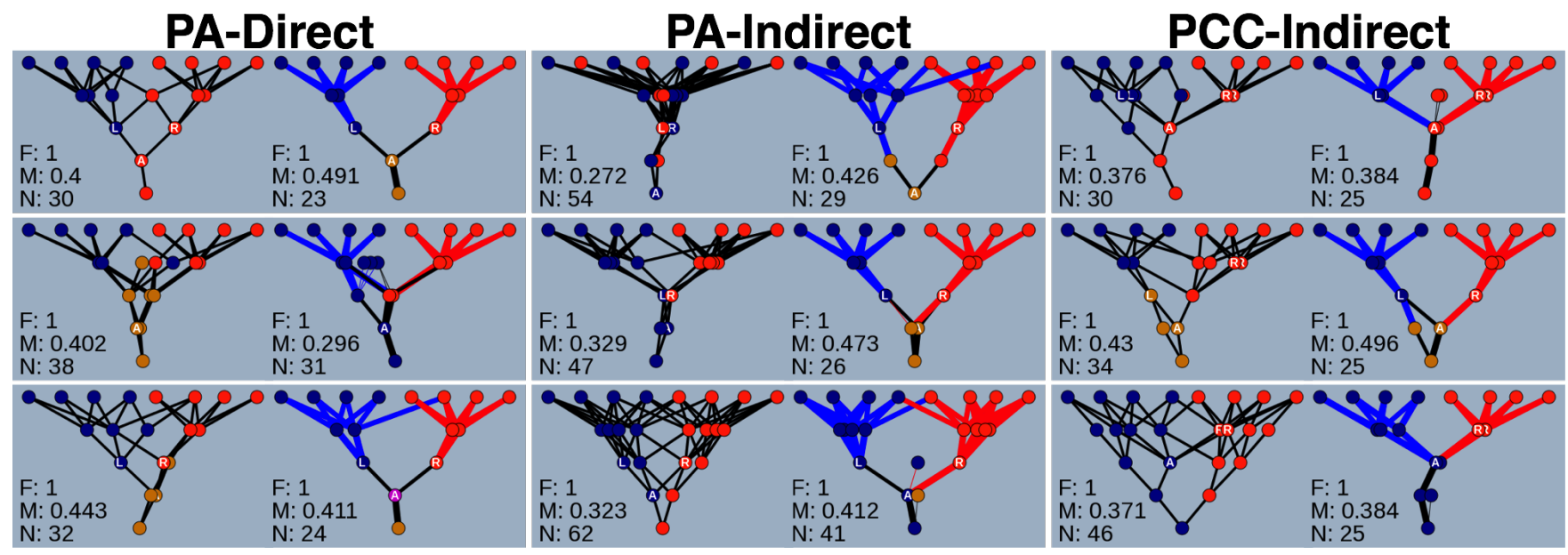

Figure 10: Functional module identification. Networks are shown in their ONP, but also with the functional module labels (connection colors) identified through SRC. The functional modules line up with the known modular decomposition of the retina problem and the targeted knockout of colored connections (blue, red, and black) produce specifics effects on retina subproblems (left-object, right-object, and AND). These two facts support that idea that these are indeed functional modules.

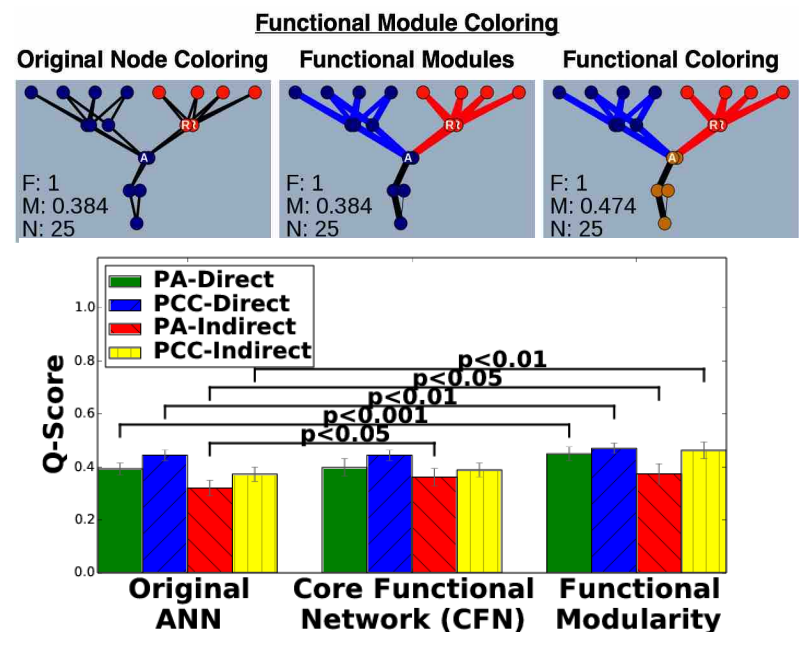

Figure 11: Q-Metric on Functional Modules The functional modules identified through SRC can be a template for placing nodes into their correct modules resulting in elevated, and more realistic Q-Scores. Top: The module labels (node colors) for many networks doesn't always put nodes into reasonable modules. When nodes are placed in modules, based on their locations within the functional modules, the modules make more sense. Bottom: Original ANN, CFN, and Functional Q-Score for all treatments. Most treatments do not see a significant increase in CFN modularity yet many CFNs look more modular. When the functional modules act as a template for the proper placement of modules the QScores for all treatments, with the exception of PA-Indirect, rises significantly $(p<0.05)$. Note, the CFN modularity of PA-Indirect is higher than it was in Figure 8. The functional module CFNs were slightly sparser and more modular than the default SRC CFNs.

\subsection{Revealing Hidden Modular Structures in Seemingly Non-Modular Networks}

SRC has revealed that hidden within the seemingly nonmodular PA-Direct, PCC-Indirect, and PA-Indirect ANNs there are indeed modular CFNs and functional modules.
Surprisingly, CFNs from these treatments are quite similar in architecture to PCC-Direct ANNs, which are arguably the ideal modular structure on this problem [6]. Three lines of evidence support this conclusion. First, a visual inspection of their CFNs reveals modular structures and functional modules similar to PCC-Direct ANNs (Figs. 5 and 10). Second, there is no significant difference between the number of connections of PCC-Direct ANNs and PA-Direct or PCCIndirect CFNs (Fig. 6; $p>0.05$ ). Third, while significant $(p<0.05)$, there is only a slight difference between the Functional Q-Score of PCC-Direct compared to PA-Direct or PCC-Indirect (Fig. 11, Bottom). All told, these lines of evidence suggest that PA-Direct networks are potentially far more similar to PCC-Direct networks functionally than was previously thought [6], especially with regards to their modularity. While less surprising, PCC-Indirect ANNs also appeared more different from PCC-Direct ANNs than their CNFs suggest they are. In contrast, the differences between PCC-Direct and PA-Indirect persists after SRC pruning.

Our discovery that some non-modular networks have hidden modular structures within them is an important revelation that can greatly contribute to the substantial scientific effort to understand modularity $[4,6,12,28]$. Recall that the ANN treatments in this study have previously been used to better understand the evolution of modularity $[6,11]$. In particular, important conclusions were made based on the differences in sparsity and modularity between PCC-Direct and PA-Direct ANNs [6]. SRC reveals that perhaps the meaningful differences between ANNs from these two treatments is smaller than previously concluded because, hidden within some seemingly non-modular ANNs, are modular solutions. That said, statistically significant differences in modularity Q-scores remain between PCC-Direct ANNs and PA-Direct CFNs. Additionally, we know from [6] that PCC-Direct networks evolve to a higher performance faster and are more evolvable than PA-Direct networks. Our work thus motivates interesting, exciting future research into what the main differences are between these treatments and why those differences contribute to different evolutionary dynamics and levels of evolvability. 


\section{FUTURE WORK AND CONCLUSION}

Our results demonstrate that $\mathrm{SRC}$ is effective at revealing core functional networks and hidden modularity in ANNs evolved for the retina problem. In future work, we will compare it to other simplification techniques and on other problems and types of neural networks. An interesting control would be to test if ANNs simplified through the removal of low magnitude weights could also produce CFNs. Regarding other domains, our preliminary results, which we will present in a future paper, suggest that SRC is similarly effective at identifying CFNs and functional modules in ANNs evolved to solve a hierarchical problems [18] and problems that require intralife learning [8]. We will also test SRC on ANNs with multiple outputs, such as those that control simulated robots [5, 15] or Deep Neural Networks trained to recognize handwritten digits [14]. With ANNs with multiple outputs, we apply SRC to each output and it finds the sub-network that is responsible for the activation of that output.

In this paper we presented SRC, a new algorithm that can simplify an ANN down to its functional core to simplify analysis. It can also identify functional modules within an ANN. When applied to ANNs evolved to solve the retina problem, a heavily explored test domain in modularity, previously hidden structures were discovered amongst ANNs thought to be non-modular, which should greatly impact ongoing research into modularity. We also showed how SRC-identified functional modules can better estimate the optimal modular decomposition of a network than state-of-the-art approaches, which improves the accuracy of the widely-used modularity Q-Score. Overall, the discovery of hidden modular structures within seemingly non-modular ANNs is an example of how SRC can improve our ability to understand ANNs, which will increase our ability to improve them.

\section{ACKNOWLEDGMENTS}

We thank Ken Gerow from the Statistics Department of the University of Wyoming for his advice on regression analysis. We also thank Joost Huizinga and Henok Mengistu of the Evolving AI Lab for their input and support. This work was funded by a NSF CAREER award (CAREER: 1453549) and a Wyoming NASA Space Grant Consortium Grant (\#NNX15AI08H).

\section{REFERENCES}

[1] M Gethsiyal Augasta and T Kathirvalavakumar. Pruning algorithms of neural networks-a comparative study. Central European Journal of Computer Science, 3(3):105-115, 2013.

[2] Y. Bengio, I. J. Goodfellow, and A. Courville. Deep learning. Book in preparation for MIT Press, 2015.

[3] Dmitri B Chklovskii. Exact solution for the optimal neuronal layout problem. Neural computation, 16(10):2067-2078, October 2004.

[4] J. Clune, B.E. Beckmann, P.K. McKinley, and C. Ofria. Investigating whether HyperNEAT produces modular neural networks. In Proc. Genetic \& Evolutionary Comput. Conf., pages 635-642. ACM, 2010.

[5] J. Clune, B.E. Beckmann, C. Ofria, and R.T. Pennock. Evolving coordinated quadruped gaits with the HyperNEAT generative encoding. In Proc. IEEE Cong. Evolutionary Comput, pages 2764-2771, 2009.

[6] J. Clune, J-B. Mouret, and H. Lipson. The evolutionary origins of modularity. Proc. Royal Society B, 280(20122863), 2013.

[7] J. Clune, K.O. Stanley, R.T. Pennock, and C. Ofria. On the performance of indirect encoding across the continuum of regularity. IEEE Trans. Evolutionary Comput, 15(4):346-367, 2011.

[8] K. O. Ellefsen, J-B. Mouret, J. Clune, and J. C. Bongard. Neural modularity helps organisms evolve to learn new skills without forgetting old skills. PLoS Comput Biol, 11(4):e1004128, 2015.

[9] T. F. Fuller, A. Ghazalpour, J. E. Aten, T. A. Drake, A. J. Lusis, and S. Horvath. Weighted gene coexpression network analysis strategies applied to mouse weight. Mammalian Genome, 18(6-7):463-472, 2007.

[10] Masafumi Hagiwara. A simple and effective method for removal of hidden units and weights. Neurocomputing, 6(2):207-218, 1994.

[11] J. Huizinga, J. Clune, and J-B Mouret. Evolving neural networks that are both modular and regular: Hyperneat plus the connection cost technique. In Proc. Genetic 8 Evolutionary Comput. Conf, pages 697-704. ACM, 2014.

[12] N. Kashtan and U. Alon. Spontaneous evolution of modularity and network motifs. Proc. Nat'l Acad. Sciences, 102(39):13773-13778, September 2005.

[13] Y. LeCun, J. S. Denker, S. A. Solla, R. E. Howard, and L. D. Jackel. Optimal brain damage. In NIPs, volume 89, 1989.

[14] Yann LeCun, Léon Bottou, Yoshua Bengio, and Patrick Haffner. Gradient-based learning applied to document recognition. Proceedings of the IEEE, 86(11):2278-2324, 1998.

[15] J. Lehman and K.O. Stanley. Abandoning objectives: Evolution through the search for novelty alone. Evolutionary Computation, 19(2):189-223, 2011.

[16] E. A. Leicht and M. E. J. Newman. Community structure in directed networks. Physical review letters, pages 118703-118707, 2008.

[17] H. Lipson. Principles of modularity, regularity, and hierarchy for scalable systems. Journal of Biological Physics and Chemistry, 7(4):125, 2007.

[18] H. Mengistu and J. Clune. The evolutionary origins of hierarchy. In preparation.

[19] A. Miller. Subset selection in regression. CRC Press, 2002.

[20] J-B Mouret and S. Doncieux. Sferes v2: Evolvin'in the multi-core world. In Evolutionary Computation (CEC), 2010 IEEE Congress on, pages 1-8. IEEE, 2010.

[21] M. E. J. Newman. Modularity and community structure in networks. Proc. Nat'l Acad. Sciences, 103(23):8577-8582, 2006.

[22] M. E. J. Newman and M. Girvan. Finding and evaluating community structure in networks. Physical review E, 69(2):026113, 2004.

[23] S. M. Smith, K. L. Miller, and et. al. Network modelling methods for fmri. Neuroimage, 54(2):875-891, 2011.

[24] O. Sporns and R. F. Betzel. Modular brain networks. Annual review of psychology, 67(1), 2015.

[25] K.O. Stanley and R. Miikkulainen. A taxonomy for artificial embryogeny. Artificial Life, 9(2):93-130, 2003.

[26] G.F. Striedter. Principles of brain evolution. Sinauer Associates Sunderland, MA, 2005.

[27] R Tibshirani. Regression shrinkage and selection via the lasso. Journal of the Royal Statistical Society. Series B (Methodological), pages 267-288, 1996.

[28] P. Verbancsics and K. O. Stanley. Constraining connectivity to encourage modularity in hyperneat. In Proc. Genetic $E_{3}$ Evolutionary Comput. Conf, pages 1483-1490. ACM, 2011.

[29] G. Wagner, M. Pavlicev, and J. M. Cheverud. The road to modularity. Nature Reviews Genetics, 8(12):921-31, December 2007.

[30] Hong-Jie Xing and Bao-Gang Hu. Two-phase construction of multilayer perceptrons using information theory. Neural Networks, IEEE Transactions on, 20(4):715-721, 2009.

[31] J. Yosinski, J.and Clune, A. Nguyen, T. Fuchs, and H. Lipson. Understanding neural networks through deep visualization. ICML Deep Learning workshop., 2015. 